\title{
Bias in food intake reporting in children and adolescents with type 1 diabetes: the role of body size, age and gender
}

\author{
Maffeis C, Schutz Y, Fornari E, Marigliano M, Tomasselli F, Tommasi M, \\ Chini V, Morandi A. Bias in food intake reporting in children and adolescents \\ with type 1 diabetes: the role of body size, age and gender. \\ Pediatric Diabetes 2017: 18: 213-221. \\ Background: An assessment of total daily energy intake is helpful in planning \\ the overall treatment of children with type 1 diabetes (T1D). However, energy \\ intake misreporting may hinder nutritional intervention. \\ Aims: To assess the plausibility of energy intake reporting and the potential \\ role of gender, body mass index (BMI) z-score (z-BMI), disease duration and \\ insulin requirement in energy intake misreporting in a sample of children and \\ adolescents with T1D. \\ Methods; The study included 58 children and adolescents aged $8-16$ yr with \\ T1D. Anthropometry, blood pressure and glycated hemoglobin (HbAlc) were \\ measured. Subjects were instructed to wear a SenseWear Pro Armband \\ (SWA) for 3 consecutive days, including a weekend day and to fill out with \\ their parents a weighed dietary record for the same days. Predicted energy \\ expenditure (pEE) was calculated by age and gender specific equations, \\ including gender, age, weight, height and physical activity level (assessed by \\ SWA). The percent reported energy intake $(\mathrm{rEI}) / \mathrm{pEE}$ ratio was used as an \\ estimate of the plausibility of dietary reporting. \\ Results: Misreporting of food intake, especially under-reporting, was \\ common in children and adolescents with T1D: more than one-third of \\ participants were classified as under-reporters and 10\% as over-reporters. Age, \\ $z$-BMI and male gender were associated with the risk of under-reporting \\ (model $\mathrm{R}^{2}=0.5$ ). Waist circumference was negatively associated with the risk \\ of over-reporting (model $\mathrm{R}^{2}=0.25$ ). \\ Conclusions: Children and adolescents with T1D frequently under-report \\ their food intake. Age, gender and z-BMI contribute to identify potential \\ under-reporters.
}

\author{
Claudio Maffeis ${ }^{\mathrm{a}}$, Yves \\ Schutz ${ }^{\mathrm{b}}$, Elena Fornari ${ }^{\mathrm{a}}$, \\ Marco Marigliano ${ }^{a}$, \\ Francesca Tomassellia, \\ Mara Tommasia, Veronica \\ Chini $^{\mathrm{a}}$ and Anita Morandi ${ }^{\mathrm{a}}$
}

aPediatric Diabetes and Metabolic Disorders Unit, University of Verona, Verona, Italy; and bepartment of Integrative Physiology, University of Fribourg, Fribourg, Switzerland

Key words: adolescent - armband children - energy expenditure energy intake - type 1 diabetes under-reporting

\author{
Corresponding author: Prof. Claudio \\ Maffeis, \\ Pediatric Diabetes and Metabolic \\ Disorders Unit, \\ University City Hospital, \\ P.le Stefani 1, \\ 37126 Verona, \\ Italy. \\ Tel: (39) 0458127662 ; \\ Fax: (39) 045 8127660; \\ e-mail: claudio.maffeis@univr.it
}

Nutrition therapy is a basic component of diabetes management (1-3). The International Society of Pediatric and Adolescent Diabetes (ISPAD) and the American Diabetes Association (ADA) recognize the importance of nutrition as an essential component of an overall healthy lifestyle, in addition to its role in controlling diabetes.

The assessment of total daily energy and nutrient intake is helpful in planning the treatment of children with type 1 diabetes (T1D) and in studying associations between dietary habits and disease complications and co-morbidities. However, in both adults and children, energy intake misreporting is common in the general population, so that information could be biased (4-16). Food intake misreporting is associated with the level of adiposity; in particular, consistent evidence is available on under-reporting of food intake in obese individuals $(4,7,9,11,12,14-18)$.

The prevalence of overweight and obesity in children and adolescents with T1D varies in the different populations but, usually, it is at least comparable if not higher than in children and adolescents without 
T1D $(19,20)$. Therefore, it is likely that also a portion of children and adolescents with T1D under-report their food intake.

To the best of our knowledge, very few data are available on the validity of energy intake reporting by children and adolescents with T1D assessed by a comparison with their daily energy expenditure (21). Therefore, the aims of this study were to assess the plausibility of energy intake reporting in a sample of children and adolescents with T1D and the relationships between gender, BMI z-score (z-BMI), disease duration and insulin requirement with energy intake reporting in this sample.

\section{Methods}

\section{Study sample}

The study included children and adolescents with T1D recruited consecutively from the diabetes clinic at the Regional Center for Pediatric Diabetes in Verona, Italy, between January 2014 and December 2014. Inclusion criteria were age range (9-16), Caucasian ethnicity, diabetes onset $>1 \mathrm{yr}$ before recruitment, T1D diagnosis confirmed by positivity for at least two among diabetes-associated autoantibodies (GADA, ZnT8A, IAA or IA-2A); exclusion criteria were chronic diseases other than T1D (except obesity), dieting, eating disorders, chronic use of drugs other than insulin, retinopathy or microalbuminuria. The children and adolescents were all following multiple dose injection (MDI) treatment with at least four injections a day, one with long-acting insulin and three or more with short-acting or regular insulin before meals or continuous subcutaneous insulin infusion (CSII). The standard nutrition education program with periodic nutrition education sessions based on ADA and ISPAD guidelines was followed $(1,2)$. Dietary education tools were selected carefully for each child and family to achieve maximum understanding and adherence. Families were provided with a reproducible meal plan with several food options that could easily be maintained.

Parents were asked to report the duration of their school education as a measure of their education level. Socio-economic status (SES) was determined based on the presence or absence of the 7RQ code on the parents' health cards, corresponding, in the Veneto region, to an annual family income $<29,000$ euros.

Of 93 eligible families, 35 did not accept to participate in the study. Children/adolescents from families who decided not to participate did not have a significantly different age $(p=0.53)$, diabetes duration $(p=0.10)$, $z$ BMI $(\mathrm{p}=0.72), \operatorname{HbA1c}(\mathrm{p}=0.38)$, gender distribution $(\mathrm{p}=0.88)$ and daily insulin requirement $/ \mathrm{kg}$ of body weight $(p=0.48)$, than those of families who accepted.
Their parents did not report any significantly different duration of school education $(\mathrm{p}=0.88$ and $\mathrm{p}=0.05$, for mothers and fathers, respectively) nor were they more likely to have an overall income $<29,000$ euros $(\mathrm{p}=0.13)$ compared with parents who participated.

\section{Detailed study protocol}

After the informed consent was signed, the children were given a physical examination to measure anthropometry and blood pressure. A venous blood sample was collected to measure HbAlc. Afterwards, the children were provided with a SenseWear Pro Armband (SWA) 6.1 (BodyMedia, Inc., Pittsburgh, PA, USA), which was positioned on the right arm. The SWA was placed on the subjects' arms for a period of $30 \mathrm{~min}$ before data collection to allow for acclimation of skin temperature. In order to obtain objectively measured data, the subjects were instructed to wear the SWA for 3 consecutive days, including a weekend day.

The children and their parents were instructed by a dietician to fill out a weighed dietary record for the $3 \mathrm{~d}$ that they wore the SWA. During the days preceding the test the children were on an unrestricted diet.

\section{Physical characteristics}

The same investigator measured height, weight, waist circumference (WC) and blood pressure, as previously reported (22). BMI was calculated as weight (in kilograms) divided by height (in meters) squared. BMI values were standardized (BMI $z$-scores) using age (to the nearest 6th month) and sex-specific median, standard deviation, and power of the BoxCox transformation (least mean square method) (23). Normal weight, overweight and obesity were defined according to the International Obesity Task Force method based on age and gender BMI cutoffs, using cut-offs from national growth charts (23). Waist-to-height ratio (WHtR) was considered a measure of body fat distribution, as previously described (24).

\section{Biochemical parameters}

HbAlc was measured using high-performance liquid chromatography with a Biorad variant 2 cation exchange column; the instrument was calibrated against Diabetes Control and Complication Trial (DCCT) approved standards.

\section{Energy intake}

A 3-d weighed dietary record of food and fluid, and the amounts consumed, was kept by the children/ adolescents and their parents $(25,26)$. Food was 
weighed on an electronic scale by the parents and, in a few instances, by one of the grandparents. Parents reported the food intake of their children at meals, and the children/adolescents were encouraged to report all the foods, including snacks, consumed outside the home. At school, every child had a personalized food tray with a defined quantity of food (first and second course, bread and fruit). For this study the kitchen supervisory staff weighed and recorded the uneaten foods on a specific sheet.

Each family was provided with a logbook for recording foods and beverages consumed. Written instructions with examples of completed forms were provided. A complete description of how the food was prepared and recipes for composite dishes were also requested. A dietician checked the logbooks with each family for completeness and accuracy on the day after the recording. As an aid to determine the amount of food and drinks consumed outside the home, pictures of different items were shown, along with cups, glasses, spoons and food shapes of different portion sizes. Food and drink energy values were calculated from tables of food comparison set out by the National Institute of Nutrition, with the use of a computerized database and analysis program (Metadieta, Meteda, S.Benedetto del Tronto, Italy) (27).

\section{Energy expenditure}

Total daily energy expenditure was predicted by ageand gender-specific equations provided by the NIH 2002 Dietary Reference Intakes (28), including weight, height and physical activity constants corresponding to physical activity level (PAL) categories. Predicted energy expenditure highly correlated with observed energy requirements from indirect calorimetry and double labeled water (29). PAL was defined as the energy expenditure/basal metabolic rate (EE/BMR) ratio, and PAL categories were: sedentary $(\mathrm{PAL} \geq 1.0<1.4)$, low active $(\mathrm{PAL} \geq 1.4<1.6)$, active $(\mathrm{PAL} \geq 1.6<1.9)$ and very active $(\mathrm{PAL} \geq 1.9<2.5)$. For each subject, the average PAL over the $3-\mathrm{d}$ period of nutritional recording was estimated from the SWA data. The SWA is a wireless multisensory body monitor worn on the triceps of the right arm. The monitor enables continuous collection of various physiological and movement parameters through multiple sensors, including an accelerometer and sensors measuring heat flux, galvanic skin response, skin temperature and near body ambient temperature. The SWA software (SENSEWEAR Professional software, version 6.1) provided measures of time spent lying down (sleeping time + non-sleep lying), corresponding to 1.0 MET expenditure rate, and time spent performing physical activities along with estimated METs associated with these active periods. The remaining time, consisting in sedentary activities, was attributed a 1.4 MET expenditure rate, based on the Recommended Daily Intake (RDI) charts (28). PAL was calculated as the weighed average METs spent every day and converted into PAL category. Predicted energy expenditure based on RDI equations including SWA-estimated PAL has been shown to be more accurate than (a) predicted energy expenditure ( $\mathrm{pEE}$ ) calculated by RDI formulas using a fixed sedentary PAL category for all children, and (b) pEE fully derived from armband equations (12).

\section{Identification of implausible food intake reporters}

On the basis of the method developed by Huang et al. (15), the percent reported energy intake/predicted energy expenditure (rEI/pEE) ratio was used as an estimate of plausibility of dietary reporting, and $\mathrm{rEI} / \mathrm{pEE}$ ratios falling under or over $100 \% \pm 1 \mathrm{SD}$ of $\mathrm{rEI} / \mathrm{pEE}$ were considered implausible: under- or overreports, respectively. In total, four standard deviations (SDs) to define plausibility ranges were calculated, in two age ranges $(9-13 \mathrm{yr}$, and $\geq 14 \mathrm{yr})$ within each gender separately, according to an equation adapted from Goldberg's cut-off calculations $(15,30)$ :

$$
1 \mathrm{SD}=\text { squared root }\left(\mathrm{CV}_{\mathrm{rEI}}^{2} / d+\mathrm{CV}_{\mathrm{pEE}}^{2}+\mathrm{CV}_{\mathrm{mTEE}}^{2}\right)
$$

where $\mathrm{CV}_{\mathrm{rEI}}$ is the sample-specific coefficient of variation in energy intake reporting, calculated by averaging individual CVs (individual $\mathrm{SD}$ of $\mathrm{rEI} /$ individual average of $3 \mathrm{~d} \mathrm{rEI}$ ); $d$ is the number of reporting days, 3 in the present study; $\mathrm{CVpEE}$ is the gender and age range-specific coefficient of variation in pEE based on the Dietary Reference Intakes data (27), for instance, 4.2 and $2.97 \%$ for 9- to 13 -yr olds and 14 to 19 -yr-old boys, respectively, and 4.81 and $4.13 \%$ for 9- to 13-yr-olds and 14- to 19-yr-old girls, respectively, as previously reported in the literature $(31,32)$, which corresponds to $8.2 \%$ for both genders and age range groups. In our sample, the value of $1 \mathrm{SD}$ was $12 \%$ in all the four gender and age range groups, so in the whole sample, participants were considered plausible reporters if their $\mathrm{rEI} / \mathrm{pEE}$ fell within the $88-112 \%$ range, probable under-reporters if it was lower than $88 \%$ and probable over-reporters if it was higher than $112 \%$. The difference between rEI and pEE was also calculated as a descriptive, clinically valuable estimate of plausibility.

Ethics statement. The protocol is in accordance with the 1975 Declaration of Helsinki as revised in 2008.

Informed consent was obtained from the children and their parents. The study was approved by the Institutional Ethics Committee of Verona (Italy). 


\section{Statistical analysis}

Results are expressed as means (SD) or proportion (percentage). Means of continuous variables were compared by the ANOVA test across the three plausibility groups (under-, plausible- and over-reporters), in case of normal distribution and variance homogeneity of variable, or by the Kruskal-Wallis test, in different cases.

Associations of energy under-reporting and overreporting with normally distributed, skewed and categorical variables were explored by comparing, respectively, variable means, rank means or proportions between under-reporters and other participants, and over-reporters and other participants, using the Student's $t$-test the Mann-Whitney test, or the $\chi^{2}$ test. Forward conditional binary logistic analyses were run to build models predicting under-reporting and over-reporting, entering variables with a p value $\leq 0.05$ in univariate analyses and removing variables showing a $p \geq 0.05$ after insertion in the multi-variate model. Continuous variables were entered as such, without any categorization.

Relationships between $\mathrm{rEI} / \mathrm{pEE}$, as a continuous estimate of reporting plausibility, and physical, biochemical and clinical variables were assessed using Pearson's correlations between rEI/pEE and the candidate continuous variables, after checking for normal distribution, and by Student's $t$-test comparison of rEI/pEE means, between groups, while considering gender and weight categories. An analysis of co-variance (ANCOVA) was done to build a model predicting $\mathrm{rEI} / \mathrm{pEE}$, using, as covariates, binary or continuous variables that were, respectively, associated or correlated with $\mathrm{rEI} / \mathrm{pEE}$ with at least a $0.05 \mathrm{p}$ value, in preliminary univariate analyses.

Differences in the principle candidate predictors (zBMI, age, gender) between under-reporters and other participants, that had an $80 \%$ probability to be detected by the convenience sample of 58 children/adolescents, were calculated (33). The sample had an $80 \%$ power to detect a 0.54 difference in z-BMI, a 2 yr difference in age and a $38 \%$ difference in male gender prevalence, between under-reporters and other participants, with a $0.05 \alpha$-error.

Data were analyzed using sPss version 20.0 software (SPSS, Chicago, IL, USA). A p value $<0.05$ was considered statistically significant.

\section{Results}

Characteristics of the sample studied are shown in Table 1. The total sample included 29 boys and 29 girls; 7 (12\%) 3 males and 4 females - were overweight, one male and one female were underweight. BMI, BMI z-scores, HbA1c, insulin requirements, parental education and SES were similar in males and females. The boys were older and had a longer T1D duration than the girls, even if these differences did not reach statistical significance. Height and SBP were significantly higher in males than in females. Girls had significantly lower rEI, PAL and pEE than boys, higher $\mathrm{rEI} / \mathrm{pEE}$ and lower (rEI-pEE) than boys (Table 1).

A total of 32 subjects $(55 \%)$ were plausible reporters whereas $6(10 \%)$ were over-reporters and $20(35 \%)$ were under-reporters (Fig. 1 and Table 2). The total sample had an average $\mathrm{rEI} / \mathrm{pEE}$ of $93 \%$, under-reporters, plausible reporters and over-reporters of 78, 99 and $116 \%$, respectively (Fig. 1).

ANOVA showed that age, z-BMI and T1D duration significantly decreased across under-, plausible- and over-reporting groups (Table 2).

Under-reporters were older and had significantly higher BMI, z-BMI, WC and systolic blood pressure, a longer duration of diabetes and higher insulin requirements than other participants (Table 2). They also included more than twice the percentage of boys (75 vs. $36 \%, p=0.012$ ) compared with other participants, and more than double the percentage of overweight (20 vs. $8 \%, \mathrm{p}=0.15)$. Overweight/obesity was associated with a 10-fold higher risk of having a $\mathrm{rEI} / \mathrm{pEE}<-2 \mathrm{DS}$, i.e., serious under-reporting - $42 \%$ compared with $3.9 \%$ among other participants, $\mathrm{p}=0.006$ (not shown). Binary logistic regression showed that a 1-yr increase in age and a unitary increase in z-BMI were associated, respectively, with 67 and $527 \%$ higher odds of energy under-reporting [odds ratio $(\mathrm{OR})=1.67(1.13-2.48)$, $\mathrm{p}=0.01 ; \mathrm{OR}=5.27(1.60-17.34), \mathrm{p}=0.006]$, whereas male gender with about a 17-fold higher odds $[\mathrm{OR}=17.24 \quad(2.52-125), \quad \mathrm{p}=0.004]$. The model explained $51 \%$ of variance in under-reporting probability $\left(\mathrm{R}^{2}\right.$ of Nagelkerke $\left.=0.508\right)$.

Over-reporters had lower BMI, z-BMI, WC and absolute insulin requirements than others (Table 2). Binary logistic regression showed that a $1 \mathrm{~cm}$ increase in WC was associated with $25 \%$ lower odds of overreporting [WC OR $=0.85(0.72-0.97), p=0.019, R^{2}$ of Nagelkerke $=0.25]$.

$\mathrm{rEI} / \mathrm{pEE}$ was inversely correlated with $\mathrm{z}$-BMI $(\mathrm{r}=-0.47, \quad \mathrm{p}<0.001)$ and WHtR $(\mathrm{r}=-0.40$, $\mathrm{p}=0.001$ ) (Fig. 2). Moreover, boys had a lower $\mathrm{rEI} / \mathrm{pEE}$ than girls [89.7 (15) vs. 97.6 (13), $\mathrm{p}<0.001]$ and overweight participants had a lower $\mathrm{rEI} / \mathrm{pEE}$ than normal weight participants [78.8 (17) vs. 95.4(13), $\mathrm{p}=0.003]$. The ANCOVA showed that $\mathrm{rEI} / \mathrm{pEE}$ was predicted by gender $[\mathrm{B}$ coefficient for males $(95 \%$ c.i. $)=-$ 12.3(-18.4; -6.08), $\mathrm{p}<0.001)$, z-BMI [B coefficient for unitary increase $=-5.2(-9.4 ;-0.96), \mathrm{p}=0.01]$ and WHtR [B coefficient for 0.01 increase $=-1.4$ $(-2.5 ;-0.2), \mathrm{p}=0.02]$, which explained 22,10 and 
Table 1. Physical characteristics, HbA1c, insulin treatment, diabetes duration, reported energy intake, physical activity level, predicted energy expenditure, parental education and socio-economic status of males and females and total sample. Data are shown as mean (standard deviation) or proportion (percentage) or median (range)

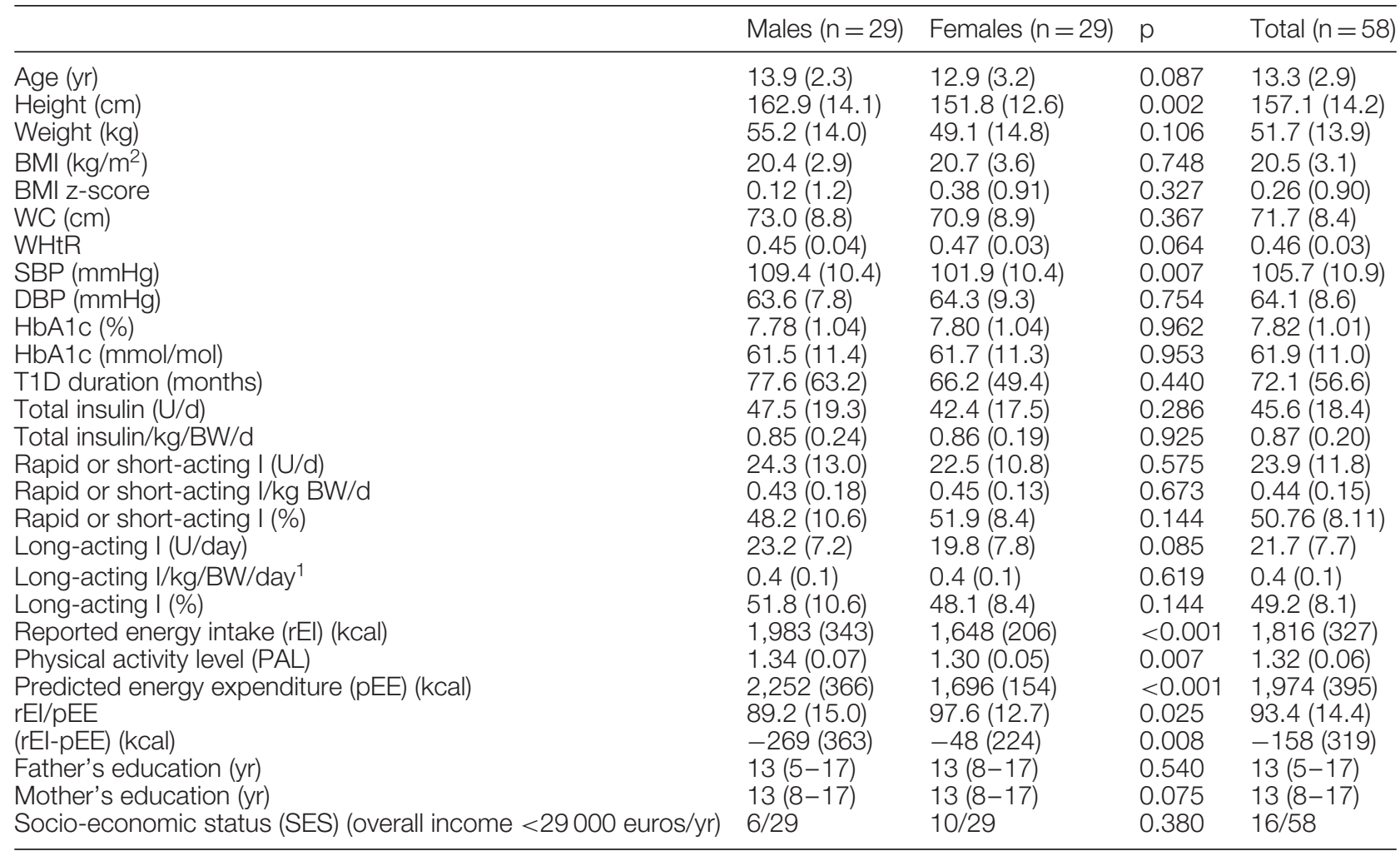

BMI, body mass index; CSII, continuous subcutaneous insulin infusion; DBP, diastolic blood pressure; HbA1c, glycated hemoglobin; MDI, multiple dose injection; rEI-pEE, reported energy intake/predicted energy expenditure; SBP, systolic blood pressure; T1D, type 1 diabetes; WHtR, waist to height ratio.

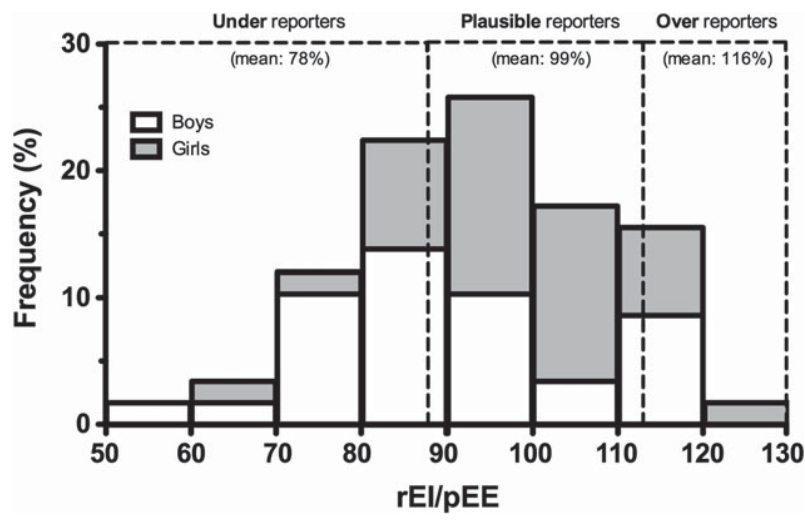

Fig. 1. Statistical distribution of the reported energy intake/predicted energy expenditure $(\mathrm{rEI} / \mathrm{pEE})$ ratio in boys $(\mathrm{n}=29)$ and girls $(\mathrm{n}=29)$.

$10 \%$ of $\mathrm{rEI} / \mathrm{pEE}$ variance, respectively, and $40 \%$ altogether.

\section{Discussion}

To the best of our knowledge, this is the second study on youth with T1D to assess the validity of food intake reporting by a comparison with total energy expenditure (21), and the first to use armband measures of physical activity, instead of questionnaires, to this purpose. The main results of this study were: (i) misreporting of food intake, especially underreporting, was common in children and adolescents with T1D; (ii) age, z-BMI and gender accounted for more than $50 \%$ of inter-individual variability of the risk of energy under-reporting; WC accounted for $25 \%$ of inter-individual variability of the risk of energy over-reporting (the lower WC, the higher the risk). WHtR, z-BMI and gender accounted for $40 \%$ of interindividual variability of $\mathrm{rEI} / \mathrm{pEE}$.

The prevalence of under-reporting is consistent with data on both healthy children and adolescents, especially data based on the Huang method to assess reporting plausibility $(15,16)$ and with those reported in the only previous study on misreporting in youth with T1D (21).

These data suggest that T1D neither increases nor decreases the risk of energy misreporting in children and adolescents. Interestingly, duration of diabetes and glycometabolic control (expressed as HbA1c) were not associated with misreporting of energy intake, further suggesting that diabetes per se does not promote a different behavior in children and their parents in regards to food intake reporting. This implies that the 
Table 2. Differences between the under-, plausible- and over-reporters. Data are shown as mean (standard deviation) or median (range)

\begin{tabular}{|c|c|c|c|c|c|c|}
\hline & $\begin{array}{l}\text { Under-reporters } \\
\qquad(\mathrm{n}=20)\end{array}$ & $\begin{array}{c}\text { Plausible } \\
\text { reporters } \\
(n=32)\end{array}$ & $\begin{array}{l}\text { Over-reporters } \\
\quad(n=6)\end{array}$ & $\begin{array}{c}\text { p between } \\
\text { groups } \\
\text { comparison }\end{array}$ & $\begin{array}{l}\text { p under-reporters } \\
\text { vs. others }\end{array}$ & $\begin{array}{l}\text { p over-reporters } \\
\text { vs. others }\end{array}$ \\
\hline Males/total & 15/20 (75) & 12/32 (37.5) & 2/6 (33.3) & 0.022 & 0.012 & 0.39 \\
\hline Age (yr) & $14.7(2.0)$ & $12.7(2.8)$ & $11.8(4.2)$ & 0.019 & 0.006 & 0.17 \\
\hline BMI $\left(\mathrm{kg} / \mathrm{m}^{2}\right)$ & $22.3(2.8)$ & $20.0(2.8)$ & $17.5(2.4)$ & 0.001 & 0.001 & 0.011 \\
\hline BMI z-score & $0.62(0.92)$ & $0.17(0.84)$ & $-0.50(0.61)$ & 0.018 & 0.025 & 0.028 \\
\hline WC (cm) & $76.3(6.6)$ & $70.5(7.7)$ & $63.2(8.7)$ & 0.001 & 0.002 & 0.008 \\
\hline WHtR & $0.47(0.04)$ & $0.45(0.03)$ & $0.44(0.02)$ & 0.23 & 0.131 & 0.25 \\
\hline $\mathrm{SBP}(\mathrm{mmHg})$ & $110.6(10.1)$ & $102.2(10.2)$ & $107.7(11.9)$ & 0.021 & 0.011 & 0.64 \\
\hline DBP (mmHg) & $63.3(7.3)$ & $63.9(9.1)$ & $68.3(10.3)$ & 0.45 & 0.596 & 0.21 \\
\hline $\mathrm{HbA1c}(\%)$ & $7.9(0.6)$ & $7.7(1.1)$ & $8.0(1.5)$ & 0.76 & 0.769 & 0.56 \\
\hline HbA1c (mmol/mol) & $62.8(6.6)$ & $61.1(12.3)$ & $64.7(16.6)$ & 0.74 & 0.764 & 0.52 \\
\hline $\begin{array}{l}\text { Duration of T1D } \\
\text { (months) }\end{array}$ & $102.7(72.5)$ & $56.4(38.4)$ & $53.7(40.1)$ & 0.009 & 0.013 & 0.40 \\
\hline CSII/total (CSII + MDI) & 4/20 (20) & 5/32 (15.6) & $1 / 6(16.6)$ & 0.92 & 0.68 & 0.97 \\
\hline Total insulin (U/d) & $57.0(15.1)$ & $41.6(16.9)$ & $29.2(17.1)$ & $<0.001$ & $<0.001$ & 0.020 \\
\hline Total insulin/kg & $0.94(0.20)$ & $0.83(0.18)$ & $0.79(0.25)$ & 0.09 & 0.030 & 0.35 \\
\hline $\begin{array}{l}\text { Rapid or short-acting } \\
\qquad \text { I (U/d) }\end{array}$ & $30.7(10.8)$ & $21.6(10.7)$ & $13.4(8.2)$ & 0.001 & 0.001 & 0.020 \\
\hline $\begin{array}{l}\text { Rapid or short acting } \\
\qquad / / \mathrm{kg}\end{array}$ & $0.50(0.15)$ & $0.42(0.13)$ & $0.36(0.12)$ & 0.049 & 0.023 & 0.15 \\
\hline $\begin{array}{l}\text { Rapid or short-acting } \\
\qquad \text { ( } \%)\end{array}$ & $52.7(6.6)$ & $50.5(9.0)$ & $46.0(6.1)$ & 0.20 & 0.196 & 0.15 \\
\hline Long-acting I (U/d) & $26.3(5.4)$ & $19.9(7.4)$ & $15.7(9.1)$ & 0.001 & 0.001 & 0.044 \\
\hline Long-acting I/kg & $0.4(0.1)$ & $0.41(0.1)$ & $0.43(0.15)$ & 0.44 & 0.256 & 0.76 \\
\hline Long-acting I (\%) & $47.3(6.6)$ & $49.5(9.0)$ & $54.0(2.5)$ & 0.20 & 0.196 & 0.15 \\
\hline $\begin{array}{l}\text { Normal weight/total } \\
\text { (\%) }\end{array}$ & 16/20 (80) & 29/32 (90.7) & $6 / 6(100)$ & 0.33 & 0.15 & 0.33 \\
\hline Overweight/total (\%) & $4 / 20(20)$ & 3/32 (9.3) & 0/6 (0) & 0.33 & 0.15 & 0.33 \\
\hline $\begin{array}{l}\text { Paternal education } \\
\text { (yr) }\end{array}$ & $8(5-17)$ & $13(8-17)$ & $13(8-17)$ & 0.21 & 0.15 & 0.88 \\
\hline $\begin{array}{l}\text { Maternal education } \\
(y r)\end{array}$ & $13(8-17)$ & $13(8-17)$ & $13(13-17)$ & 0.21 & 0.28 & 0.18 \\
\hline $\begin{array}{l}\text { Family SES (overall } \\
\text { income <29000 } \\
\text { euros/yr) }\end{array}$ & $4 / 20$ & $11 / 32$ & $1 / 6$ & 0.43 & 0.53 & 0.88 \\
\hline
\end{tabular}

BMI, body mass index; CSII, continuous subcutaneous insulin infusion; DBP, diastolic blood pressure; HbA1c, glycated hemoglobin; MDI, multiple dose injection; rEl/pEE, reported energy intake/predicted energy expenditure; SBP, systolic blood pressure; SES, socio-economic status; T1D, type 1 diabetes; WC, waist circumference; WHtR, waist to height ratio.

nutrition education program we offered to children and adolescents and their families was not enough to raise adequate consciousness on food intake. Further actions in promoting family-based meal times and meal structure might be necessary to improve food intake awareness. Moreover, counseling strategies designed to break down reluctance to admitting one's over-eating, another plausible reason for under-reporting, could also be helpful, especially among overweight patients. The inverse relationship between z-BMI and $\mathrm{rEI} / \mathrm{pEE}$ is in agreement with a large amount of previous evidence on non-diabetic children and adolescents $(4,7,9,11$, $12,14-18)$, but it is in contrast with the only abovementioned previous study of youth with T1D, which did not find any association between energy intake reporting and adiposity (21). It is noteworthy that our sample had a low prevalence of overweight/obesity $(12 \%)$, in line with that observed and discussed in previous studies assessing cardiovascular risk factors in an independent sample of children/adolescents with T1D recruited in the same area (22), and a much lower prevalence than that reported in the above-cited study (34\%) (21).

This may imply profound differences in the average nutritional consciousness and behavior as well as in the average willingness to admit one's energy intake, between the two studied populations. This may in turn imply different roles of adiposity in affecting energy reporting.

Under-reporting was predicted by z-BMI and overreporting by WC. Both z-BMI and WHtR independently accounted for $10 \%$, each, of the variability in $\mathrm{rEI} / \mathrm{pEE}$ considered as a continuous variable. This suggests that both total adiposity and its distribution influence the plausibility of energy reporting. A potential explanation of this finding may be that the higher 

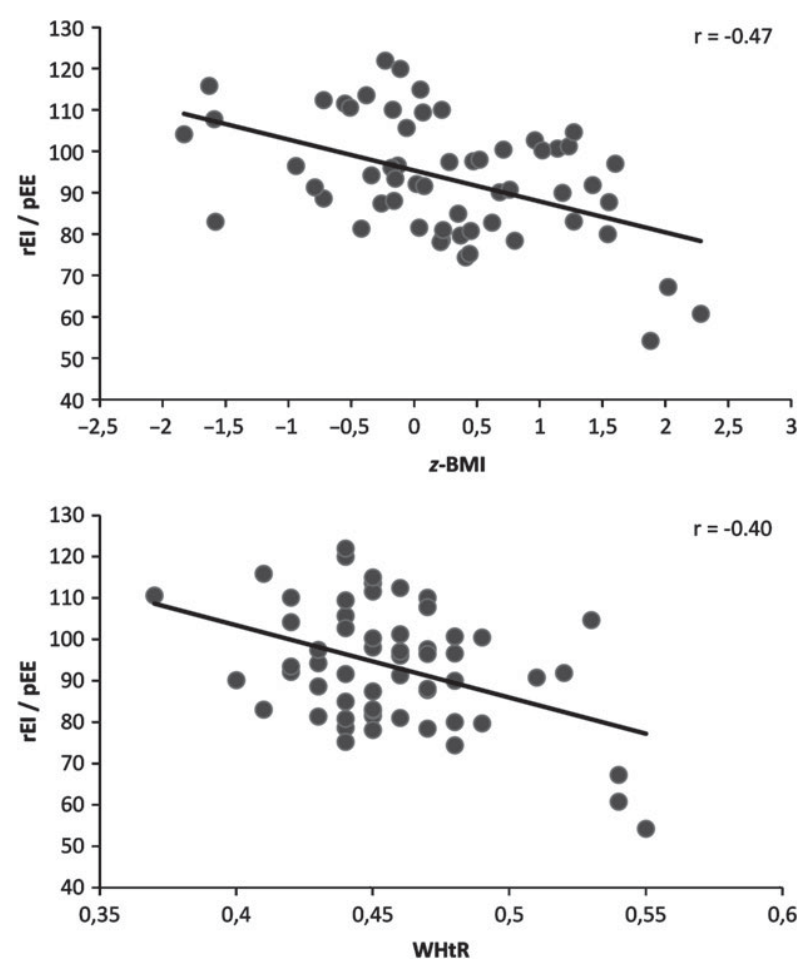

Fig. 2. Inverse relationship between the reported energy intake/ predicted energy expenditure (rEI/pEE) ratio and body mass index (BMI) (expressed as $z$-scores) and waist-to-height ratio in the total sample.

fat accumulation in the abdominal area may induce a higher concern of one's own adiposity and, by implication, to encourage a lower self-reporting of the food actually ingested. Consistently, a relationship between weight concern and under-reporting of food intake was previously showed in children and adolescents $(4,14,16)$.

Interestingly, in our sample, boys were three times more likely to under-report energy intake than girls, but the average magnitude of under-reporting was modest (11\% in boys vs. about $3 \%$ in girls). This was independent of z-BMI and age, and, moreover, gender explained the same percentage of inter-individual variability in $\mathrm{rEI} / \mathrm{pEE}$ as $\mathrm{z}$-BMI and WHtR taken together. To the best of our knowledge, this is not consistent with previous evidence on a gender effect on energy reporting in children. Of 13 studies assessing gender, 3 found significant differences, 2 with females more likely to under-report energy intake, and 1 with males modestly more likely to do the same $(10,14)$. It is not easy to hypothesize plausible explanations for this important gender effect, which could arise from intrinsic male gender-associated difficulties in healthy nutrition and plausible dietary reporting in populations with T1D, or possibly from unconsciously unequal or not equally effective nutrition education provided to boys and girls and their families by our team. A potential systematic over-estimation of energy expenditure in males compared with females by the method adopted in this study is unlikely on the basis of objectively assessed minutes of physical activity by Armband instead of self-reported data on a questionnaire as performed in other studies (14). Nevertheless, further studies are needed to explore the role of gender on energy reporting and factors modulating this role in this age group.

Ten percent of the total sample over-reported their energy intake and had a z-BMI ranging from -2 to 0 (average: -0.50 ). This suggests that some children/adolescents with T1D could be concerned with food intake and overestimate it even in absence of an overt restrictive weight disorder. Longitudinal studies are warranted to assess whether energy over-reporting is a predictor of energy restriction in patients with T1D.

Our study has some potential limitations: (i) the studied sample, although $\geq 80 \%$ powerful to detect the reported associations of gender, z-BMI and age with misreporting, was too small to provide accurate estimates of the effect size of these variables, whose ORs display, in fact, large confidence intervals. Therefore, further studies with a larger sample size may help to reduce the uncertainty concerning the effect sizes; (ii) the cut-off points used in this paper for the degree of energy intake plausibility may be challenged, although the approach presented has been used by other investigators $(15,16)$. In fact, it may be argued that the $\pm 1 \mathrm{SD}$ of $\mathrm{pEI} / \mathrm{pEE}$ interval could be ineffective in separating children who really under-reported energy intake for inherent measurement error or behavioral factors from those who simply had a lower energy intake than predicted, during the 3 -d recording period, because of a 'natural' variation in the day-to-day energy intake in human subjects. Nevertheless, in order to validate our approach, we calculated the intraindividual day-by-day variation (CV) of energy intake in our sample, which was on average $10.5 \%$ (6.2) for males and $11.8 \%(6.2)$ for females. These values are very close to the SD stated in the formula we adopted to assess the plausibility interval (12\%), which reinforces the formula's reliability. (iii) The lack of a control group. However, the aim of the study was to assess differences in reporting energy intake in a sample of children and adolescents with T1D and not to compare subjects with and without diabetes; (iv) ethnicity. The results of our study on a sample of children of European ancestry may not be directly extended to children of other ethnic groups without further studies; (v) the method used to assess EI and EE. Previous studies showed that all the methods available for measuring energy intake in children and adolescents in freeliving conditions show a certain level of inaccuracy. The weighed dietary method we used in this study proved to be fairly accurate from a comparison with total energy expenditure measured by the doubly 
labeled water (DLW) method (13). To estimate total energy expenditure we predicted EE by RDI equations including physical activity coefficients, which proved to be reasonably accurate in comparison with energy expenditure measured by indirect calorimetry and the DLW (31). Obviously, the use of the DLW method might be preferable. However, high costs, difficulty of ${ }^{18} \mathrm{O}$ availability in the world, and technical complexity for isotope measurements limit the use of the DLW method in a clinical setting. Therefore, considering the purposes of this study, the use of dietary recording and RDI equations for $\mathrm{pEE}$ can be considered fairly accurate.

The study also has several points of strength: (i) the simultaneous measure of EI and EE in free-living conditions, with the use of SWA to assess PAL more accurately than by 'guesstimate'; (ii) the simultaneous exploration of several potential predictors of energy misreporting, among which disease-related variables.

In conclusion, children and adolescents with T1D tend to report inadequately their energy intake - in particular to under-report. Under-reporting is positively associated with z-BMI, age and male gender. These findings suggest that clinicians must be cautious in trusting the energy intake data reported by children and adolescents with T1D. In practical terms, a greater effort should be devoted to increasing the food intake awareness of children and adolescents with T1D.

\section{Acknowledgements}

The study was funded by the University of Verona, Department of Life \& Reproduction Sciences (University Funds for Research FUR \# 2013 MAFF).

We heartily thank the patients and their families who participated with enthusiasm in the study.

\section{References}

1. Smart Ce, Annan F, Bruno LP, Higgins LA, ACERINI CL. International Society for Pediatric and Adolescent Diabetes. ISPAD Clinical Practice Consensus Guidelines 2014. Nutritional management in children and adolescents with diabetes. Pediatr Diabetes 2014: 15 (Suppl. 20): 135-153.

2. American Diabetes Association. Standards of medical care in diabetes-2012. Diabetes Care 2012: 35 (Suppl. 1): S11-S63.

3. Maffeis C, Pinelli L. Teaching children with diabetes about adequate dietary choices. Br J Nutr 2008: 99: S33-S39.

4. Farajian P, Bountziouka V, Risvas G, Panagiotakos DB, Zampelas A. Anthropometric, lifestyle and parental characteristics associated with the prevalence of energy intake misreporting in children: the GRECO (Greek Childhood Obesity) study. Br J Nutr 2015: 113: $1120-1128$.

5. Hare ME, Sherrill-Mittleman D, Klesges RC, LANCTOT JQ, KLESGES LM. Energy under-reporting in
African-American girls: a longitudinal analysis. Child Obes 2012: 8: 551-560.

6. Rangan AM, Flood VM, Gill TP. Misreporting of energy intake in the 2007 Australian Children's Survey: identification, characteristics and impact of misreporters. Nutrients 2011: 3: 186-199.

7. Lanctot JQ, Klesges RC, Stockton MB, Klesges LM. Prevalence and characteristics of energy underreporting in African-American girls. Obesity (Silver Spring) 2008: 16: 1407-1412.

8. Stice E, Durant S. Elevated objectively measured but not self-reported energy intake predicts future weight gain in adolescents. Appetite 2014: 81: 84-88.

9. Murakami K, Miyake Y, Sasaki S, Tanaka K, Arakawa M. Characteristics of under- and overreporters of energy intake among Japanese children and adolescents: The Ryukyus Child Health Study. Nutrition 2012: 28: 532-538.

10. Kobe H, KRŽIŠNiK C, Mis NF. Under- and overreporting of energy intake in slovenian adolescents. J Nutr Educ Behav 2012: 44: 574-583.

11. Lioret S, Touvier M, Balin M et al. Characteristics of energy under-reporting in children and adolescents. $\mathrm{Br}$ J Nutr 2011: 105: 1671-1680.

12. Noel SE, Mattocks C, Emmett P, Riddoch CJ, Ness AR, Newby PK. Use of accelerometer data in prediction equations for capturing implausible dietary intakes in adolescents. Am J Clin Nutr 2010: 92: 1436-1445.

13. Burrows Tl1, Martin RJ, Collins CE. A systematic review of the validity of dietary assessment methods in children when compared with the method of doubly labeled water. J Am Diet Assoc 2010: 110: 1501-1510.

14. Forrestal SG. Energy intake misreporting among children and adolescents: a literature review. Matern Child Nutr 2011: 7: 112-127.

15. Huang TT, Howarth NC, Lin BH, Roberts SB, MCCRORY MA. Energy intake and meal portions: associations with BMI percentile in U.S. children. Obes Res 2004: 12: 1875-1885.

16. Ventura AK, Loken E, Mitchell DC, SmiciklasWright H, BIRCH LL. Understanding reporting bias in the dietary recall data of 11-year-old girls. Obesity (Silver Spring) 2006: 14: 1073-1084.

17. Nielsen BM, Nielsen MM, Toubro $S$ et al. Past and current body size affect validity of reported energy intake among middle-aged Danish men. J Nutr 2009: 139: 2337-2343

18. MAFFeIs C. Food intake and childhood obesity: accurate estimation of requirements? Lancet Diabetes Endocrinol 2013: 1: 80-1.

19. Dubose SN, Hermann JM, Tamborlane WV et al. Type 1 diabetes exchange clinic network and diabetes prospective follow-up registry. obesity in youth with type 1 diabetes in Germany, Austria, and the United States. J Pediatr 2015: 167: 627-32.e1-4.

20. Pinhas-Hamiel O, Levek-Motola N, Kaidar K et al. Prevalence of overweight, obesity and metabolic syndrome components in children, adolescents and young adults with type 1 diabetes mellitus. Diabetes Metab Res Rev 2015: 31: 76-84.

21. Sands Al, Higgins LA, Mehta SN, Nansel TR, LIPSKY LM, LAFFEL LM. Associations of youth and parent weight status with reported versus predicted daily energy intake and hemoglobin A1c in youth with 
type 1 diabetes mellitus. J Diabetes Sci Technol 2013: 7: $263-270$.

22. Maffeis C, Morandi A, Ventura E et al. Diet, physical, and biochemical characteristics of children and adolescents with type 1 diabetes: relationship between dietary fat and glucose control. Pediatr Diabetes 2012: 13: $137-146$.

23. Cacciari E, Milani S, Balsamo A et al. Italian crosssectional growth charts for height, weight and BMI (2 to $20 \mathrm{yr}$ ). J Endocrinol Invest 2006: 29: 581-593.

24. Morandi A, Miraglia Del Giudice E, Martino F, Martino E, Bozzola M, Maffeis C. Anthropometric indices are not satisfactory predictors of metabolic comorbidities in obese children and adolescents. J Pediatr 2014: 165: 1178-1183.

25. Livingstone MB, Prentice AM, Coward WA et al. Validation of estimates of energy intake by weighed dietary record and diet history in children and adolescents. Am J Clin Nutr 1992: 56: 29-35.

26. Walter Willet. 24-Hour Dietary Recall and Food Record Method. Nutritional Epidemiology. 1998. DOI:10.1093/acprof:oso/9780195122978.003.04.

27. National Institute for the Research in Food and Nutrition. Food composition tables. XXX: Edra Publ., 2007.
28. Institute of Medicine. Dietary Reference Intakes for Energy, Carbohydrate, Fiber, Fat, Fatty Acids, Cholesterol, Protein, and Amino Acids, Part I. Academy of Sciences. Washington, DC: The National Academic Press, 2002.

29. Goldberg GR, Black AE, JebB SA et al. Critical evaluation of energy intake data using fundamental principles of energy physiology: 1. derivation of cut-off limits to identify under-recording. Eur J Clin Nutr 1991: 45: 569-581.

30. BLACK AE. Critical evaluation of energy intake using the Goldberg cut-off for energy intake: basal metabolic rate. A practical guide to its calculation, use and limitations. Int J Obes Relat Metab Disord 2000: 24: 1119-1130.

31. McCrory MA, Hajduk CL, Roberts SB. Procedures for screening out inaccurate reports of dietary energy intake. Public Health Nutr 2002: 5 (6A): 873-882.

32. BlaCK AE, COLE TJ. Within- and between-subject variation in energy expenditure measured by the doublylabelled water technique: implications for validating reported dietary energy intake. Eur J Clin Nutr 2000: 54: 386-394.

33. Bernard RosNer. Fundamentals of Biostatistics 5th edition. Thomson Brooks/Cole, 1999. ISBN: 97805343 70688 . 